\title{
FCGR2A Promoter Methylation and Risks for Intravenous Immunoglobulin Treatment Responses in Kawasaki Disease
}

\author{
Ho-Chang Kuo, ${ }^{1}$ Yu-Wen Hsu, ${ }^{2}$ Mei-Shin Wu, ${ }^{2}$ Peng Yeong Woon, ${ }^{3}$ \\ Henry Sung-Ching Wong, ${ }^{4}$ Li-Jen Tsai, ${ }^{5}$ Ruo-Kai Lin, ${ }^{6}$ Sukhontip Klahan, ${ }^{2}$ \\ Kai-Sheng Hsieh, ${ }^{1}$ and Wei-Chiao Chang ${ }^{2,4,7,8}$ \\ ${ }^{1}$ Department of Pediatrics and Kawasaki Disease Center, Kaohsiung Chang Gung Memorial Hospital and \\ Chang Gung University College of Medicine, Kaohsiung 80031, Taiwan \\ ${ }^{2}$ Department of Clinical Pharmacy, College of Pharmacy, Taipei Medical University, Taipei 11696, Taiwan \\ ${ }^{3}$ Department of Molecular Biology and Human Genetics, Tzu Chi University, Hualien 97004, Taiwan \\ ${ }^{4}$ Master Program for Clinical Pharmacogenomics and Pharmacoproteomics, School of Pharmacy, Taipei Medical University, \\ Taipei 11696, Taiwan \\ ${ }^{5}$ Graduate Institute of Medical Sciences, College of Medicine, Taipei Medical University, Taipei 11696, Taiwan \\ ${ }^{6}$ Graduate Institute of Pharmacognosy, Taipei Medical University, Taipei 11696, Taiwan \\ ${ }^{7}$ Graduate Institute of Clinical Medicine, College of Medicine, Kaohsiung Medical University, Kaohsiung 80031, Taiwan \\ ${ }^{8}$ Department of Pharmacy, Taipei Medical University-Wan Fang Hospital, Taipei 11696, Taiwan
}

Correspondence should be addressed to Wei-Chiao Chang; wcc@tmu.edu.tw

Received 21 July 2014; Accepted 7 October 2014

Academic Editor: Shengyu Yang

Copyright (c) 2015 Ho-Chang Kuo et al. This is an open access article distributed under the Creative Commons Attribution License, which permits unrestricted use, distribution, and reproduction in any medium, provided the original work is properly cited.

\begin{abstract}
Kawasaki disease (KD) is characterized by pediatric systemic vasculitis of an unknown cause. The low affinity immunoglobulin gamma Fc region receptor II-a (FCGR2A) gene was reported to be involved in the susceptibility of KD. DNA methylation is one of the epigenetic mechanisms that control gene expression; thus, we hypothesized that methylation status of CpG islands in FCGR2A promoter associates with the susceptibility and therapeutic outcomes of Kawasaki disease. In this study, 36 KD patients and 24 healthy subjects from out-patient clinic were recruited. Eleven potential methylation sites within the targeted promoter region of FCGR2A were selected for investigation. We marked the eleven methylation sites from A to K. Our results indicated that methylation at the $\mathrm{CpG}$ sites $\mathrm{G}, \mathrm{H}$, and J associated with the risk of $\mathrm{KD}$. CpG sites B, C, E, F, H, J, and $\mathrm{K}$ were found to associate with the outcomes of IVIG treatment. In addition, CpG sites G, J, and $\mathrm{K}$ were predicted as transcription factors binding sites for NF-kB, Myc-Max, and SP2, respectively. Our study reported a significant association among the promoter methylation of FCGR2A, susceptibility of $\mathrm{KD}$, and the therapeutic outcomes of IVIG treatment. The methylation levels of CpG sites of FCGR2A gene promoter should be an important marker for optimizing IVIG therapy.
\end{abstract}

\section{Introduction}

Kawasaki disease (KD) is characterized as an acute systemic vasculitic syndrome [1]. It is recognized as a worldwide disease and is thought to be the leading cause of acquired heart disease in children in developed countries [2-4]. Intravenous immunoglobulin (IVIG) is the standard treatment for KD patients. Receiving high dosage of IVIG within seven days after disease onset could reduce the risk of coronary artery damage significantly.
The etiology of Kawasaki disease (KD) remains unclear. However, the epidemiological studies indicated that the prevalence of KD is higher in East Asia than Europe [5]. Uehara et al. suggested that patients with KD family medical history had higher incidence rate than others [6]. Therefore, genetic factor is considered as an important factor in the pathogenesis of $\mathrm{KD}$. Indeed, genome-wide association study (GWAS) and genetics studies have implicated several susceptibility loci for KD [7-15]. Among these studies, the FCGR2A was found as one of the important genetic factors. 
TABLE 1: Basal characteristics of patients with Kawasaki disease (KD) and normal controls.

\begin{tabular}{lcc}
\hline Characteristics & KD patients & Control subjects \\
\hline Number of subjects & $36^{\dagger}$ & 24 \\
Gender: male (\%) & $25(69.4 \%)$ & $11(45.8 \%)$ \\
Age (years) & $1.57 \pm 1.21$ & $4.91 \pm 2.78$ \\
Age range & $0-5$ & $1-11$ \\
\hline
\end{tabular}

${ }^{\dagger}$ The number included 13 IVIG-nonresponsive and 22 IVIG-responsive patients and $1 \mathrm{KD}$ patient without record of using IVIG treatment.

A functional polymorphism of FCGR2A elevated the risk of KD [11].

Several lines of evidence have indicated the strong association between methylation status of gene promoter regions and diseases. For example, the $\mathrm{CpG}$ methylation pattern of the proximal insulin gene promoter was strongly associated with type 1 diabetes (T1D) in a French T1D cohort [16]. BRCA1 promoter methylation on rs11655505 (c.2265C>T) variants was also reported to associate with sporadic breast cancer [17]. In this study, we designed experiments to investigate the association between methylation status of $\mathrm{CpG}$ islands on FCGR2A promoter and the clinical outcomes of IVIG therapy in Kawasaki disease.

\section{Materials and Methods}

2.1. Patients and Healthy Controls. We recruited $36 \mathrm{KD}$ patients from out-patient clinic and 24 healthy subjects from out-patient clinic in this study (Table 1). KD patients fulfilled the diagnostic criteria as described in our previous studies [12-15]. This study was approved by Institutional Review Board of Kaohsiung Chang Gung Memorial Hospital. Informed consent was obtained from all individuals' parents or guardians before any test was conducted.

2.2. Evaluation of IVIG Responsiveness. IVIG-responsiveness was defined as defervescence within 48 hours after initial IVIG and no reappearance of fever $\left(>38^{\circ} \mathrm{C}\right)$ for at least 7 days [18]. A standard IVIG treatment was employed and the details of the procedures were described previously [18].

2.3. FCGR2A Promoter CPG Methylation Analysis. Bisulfite sequencing technology was performed by Pyromark Q24 machine to profile the methylation levels on the FCGR2A promotor region within our targeted 500 bps range (1q23.3; chr1:161,474,603-161,475,102). In our target region, eleven CpG methylation loci were predicted by Pyromark Q24 software (Qiagen Inc., Valencia, CA, USA) and we marked the sites according to the order of English alphabet.

2.4. Transcription Factors Binding Sites Prediction. JASPAR, an open-access database, was applied to predict the potential transcription factor binding sites (TFBSs) [19]. The motifs of candidate TFBSs were assigned scores by the position weight matrix for the given sequence. The scoring algorithm was reviewed by Stormo GD in year 2000 [20]. Each binding model of the score range has their own uniqueness and cannot be compared directly; therefore, JASPAR normalized these ranges and was displayed as relative matrix score. The method for determining the relative matrix score was described by Lenhard et al. [21]. The database only listed the TFs which have the relative matrix scores above 0.80 .

2.5. Statistical Analysis. Statistical analysis was performed using SPSS, version 14.0 (SPSS Int., Chicago, Illinois, USA). The difference of methylation levels between patient and control groups was analyzed by the Chi-square $\left(\chi^{2}\right)$ test.

\section{Results}

3.1. Characteristics of KD Patients and Normal Controls. 60 samples were collected in this study, including $36 \mathrm{KD}$ patients and 24 healthy subjects. The information of age and sex ratio for $\mathrm{KD}$ patients and control subjects is shown in Table 1. In the KD group, $13 \mathrm{KD}$ patients were nonresponsive to intravenous immunoglobulin (IVIG) treatment, whereas $22 \mathrm{KD}$ patients were responsive to IVIG therapy and $1 \mathrm{KD}$ patient was without IVIG therapy record.

3.2. Methylation Status of FCGR2A Promoter Region. Both bisulfite sequencing results and clinical data were combined to investigate the association between $\mathrm{KD}$ susceptibility and methylation levels. As shown in Table 2, we found that the increased methylation levels at three of the eleven studied CpG sites associated with KD susceptibility. Among the three $\mathrm{CpG}$ sites, a strong association was found between methylation of $\mathrm{CpG}$ site $\mathrm{J}$ and susceptibility of Kawasaki disease $(P=0.0003)$. In addition, increased methylations at the $\mathrm{CpG}$ site $\mathrm{G}$ and site $\mathrm{H}$ also correlated with the risk of Kawasaki disease $(P=0.0038$ and $P=0.0019$, resp.).

3.3. Pharmacogenomic Analysis for IVIG Therapy. In the treatment of $\mathrm{KD}, I V I G$ is one of the major medications. However, some patients were nonresponsive to IVIG $(2 \mathrm{~g} / \mathrm{kg})$ treatment. We, therefore, further tested whether the promoter methylation status of FCGR2A associated with the IVIG treatment outcomes. As shown in Table 3, increased methylation levels on CpG sites B, E, F, H, and J strongly associated with the nonresponsive patients to IVIG $(P<0.0001)$.

3.4. Bioinformatics Analysis for Potential Binding Sites. To understand the possible mechanisms of $\mathrm{CpG}$ sites, online transcription factor binding site prediction program, JASPAR, was applied to predict the candidate transcription factors (TFs). Among the eight TFs, NF-kB, Myc-Max, and SP2 were significantly predicted to bind to the area that covered at least one base of the $\mathrm{CpG}$ methylation sites (Table 4).

\section{Discussion}

Methylation and demethylation of cytosine residues in the promoter regions play a crucial role in many vital biological processes. Disease manifestation due to genomic imprinting 
TABLE 2: Means and standard deviations of the percentage of methylation detected at each CpG site in KD patients and normal controls.

\begin{tabular}{|c|c|c|c|c|c|c|c|c|}
\hline \multirow{3}{*}{ Gene } & \multicolumn{8}{|c|}{ Methylation (\%) } \\
\hline & \multirow[t]{2}{*}{ CpG sites } & \multicolumn{3}{|c|}{ KD patients $(N=36)$} & \multicolumn{3}{|c|}{ Control subjects $(N=24)$} & \multirow[t]{2}{*}{$P$ value } \\
\hline & & Mean & $\mathrm{SD}$ & $95 \% \mathrm{CI}$ & Mean & $\mathrm{SD}$ & $95 \% \mathrm{CI}$ & \\
\hline \multirow{11}{*}{$F C G R 2 A$} & A & 80.61 & 5.06 & $78.90-82.33$ & 78.92 & 4.42 & $77.05-80.78$ & 0.1856 \\
\hline & $\mathrm{B}$ & 62.75 & 9.51 & $59.54-65.97$ & 62.31 & 5.13 & $60.15-64.48$ & 0.8372 \\
\hline & $\mathrm{C}$ & 70.16 & 5.61 & $68.26-72.06$ & 70.22 & 6.26 & $67.57-72.86$ & 0.9711 \\
\hline & $\mathrm{D}$ & 67.82 & 5.10 & $66.10-69.55$ & 66.49 & 4.69 & $64.51-68.47$ & 0.3112 \\
\hline & $\mathrm{E}$ & 61.51 & 5.87 & $59.52-63.49$ & 59.84 & 3.57 & $58.33-61.35$ & 0.2175 \\
\hline & $\mathrm{F}$ & 82.16 & 7.24 & $79.71-84.61$ & 80.24 & 5.87 & $77.76-82.72$ & 0.2852 \\
\hline & G & 68.66 & 5.43 & $66.82-70.49$ & 64.36 & 5.35 & $62.10-66.62$ & $0.0038^{* *}$ \\
\hline & $\mathrm{H}$ & 43.19 & 11.83 & $39.19-47.20$ & 34.92 & 4.55 & $33.00-36.84$ & $0.0019^{* *}$ \\
\hline & I & 83.74 & 5.75 & $81.79-85.68$ & 86.56 & 6.73 & $83.72-89.40$ & 0.0874 \\
\hline & J & 62.30 & 8.20 & $59.52-65.07$ & 55.12 & 4.98 & $53.01-57.22$ & $0.0003^{* * *}$ \\
\hline & $\mathrm{K}$ & 67.54 & 7.19 & 65.11-69.98 & 66.86 & 5.51 & $64.53-69.18$ & 0.6982 \\
\hline
\end{tabular}

The statistical significance ${ }^{* *} P<0.01,{ }^{* * *} P<0.001$.

TGCAAGCTCTGCCTCCCG ${ }^{\mathrm{A}}$ GGTTCACG $^{\mathrm{B}}$ CCATTCTCCTGCCTCAGCCTCCCG ${ }^{\mathrm{C}}$ AGTAGCTGGGACTATCTGCCACCG ${ }^{\mathrm{D}} \mathbf{C G}^{\mathrm{E}} \mathrm{CCCG}^{\mathrm{F}}$ GCTAAATTTTTTTTGTATTATTAGTAGAGACG ${ }^{\mathrm{G}}$ GGGTTTCACCG $^{\mathrm{H}}$ TGTTAGCCAGGATGGTCTCG $^{\mathrm{I}}$ ATCTCCTGACCTCG $^{\mathrm{J}}$ TGATCCACCCG $^{\mathrm{K}}$ CCTTGGCCTCCCAAAG.

TABle 3: Means and standard deviations of the percentage of methylation detected at each CpG site in KD patients responding or not responding to intravenous immunoglobulin treatment.

\begin{tabular}{|c|c|c|c|c|c|c|c|c|}
\hline \multirow{3}{*}{ Gene } & \multicolumn{8}{|c|}{ Methylation (\%) } \\
\hline & \multirow[t]{2}{*}{ CpG sites } & \multicolumn{3}{|c|}{ IVIG-nonresponsive patients $(N=13)$} & \multicolumn{3}{|c|}{ IVIG-responsive patients $(N=22)$} & \multirow[t]{2}{*}{$P$ value } \\
\hline & & Mean & SD & $95 \% \mathrm{CI}$ & Mean & SD & $95 \% \mathrm{CI}$ & \\
\hline \multirow{11}{*}{ FCGR2A } & A & 80.06 & 4.51 & $77.33-82.79$ & 80.94 & 5.55 & $78.48-83.40$ & 0.6319 \\
\hline & $\mathrm{B}$ & 71.40 & 6.65 & $67.39-75.42$ & 57.55 & 7.11 & $54.40-60.70$ & $<0.0001^{* * *}$ \\
\hline & $\mathrm{C}$ & 72.93 & 5.64 & $69.52-76.34$ & 68.40 & 5.09 & $66.15-70.66$ & $0.0201^{*}$ \\
\hline & $\mathrm{D}$ & 69.46 & 4.72 & $66.60-72.31$ & 66.85 & 5.28 & $64.50-69.19$ & 0.1516 \\
\hline & $\mathrm{E}$ & 67.65 & 4.43 & $64.97-70.33$ & 57.90 & 3.03 & $56.56-59.25$ & $<0.0001^{* * *}$ \\
\hline & $\mathrm{F}$ & 88.34 & 4.92 & $85.37-91.31$ & 78.47 & 5.98 & $75.82-81.12$ & $<0.0001^{* * *}$ \\
\hline & G & 68.38 & 5.71 & $64.93-71.83$ & 68.95 & 5.48 & $66.52-71.38$ & 0.7723 \\
\hline & $\mathrm{H}$ & 55.32 & 9.42 & $49.63-61.02$ & 36.38 & 6.33 & $33.57-39.19$ & $<0.0001^{* * *}$ \\
\hline & I & 83.56 & 5.59 & $80.18-86.94$ & 83.48 & 5.83 & 80.89-86.06 & 0.9650 \\
\hline & $\mathrm{J}$ & 69.26 & 6.72 & $65.19-73.32$ & 58.53 & 6.22 & $55.77-61.29$ & $<0.0001^{* * *}$ \\
\hline & $\mathrm{K}$ & 71.30 & 6.92 & $67.12-75.48$ & 65.27 & 6.68 & $62.31-68.24$ & $0.0158^{*}$ \\
\hline
\end{tabular}

The statistical significance ${ }^{*} P<0.05,{ }^{* * *} P<0.0001$.

such as Angelman syndrome and Prader-Willi syndrome are two of the classical examples of genomic DNA methylation involving on human chromosomal region 15q11-13 [22]. In addition, it has been reported that, in fulminant type 1 diabetes, DNA methylation within the Foxp3 promoter impaired TLR9-induced FOXP3 expression by attenuating IRF-7 binding activity [23]. FCGR2A is the most widely expressed $I g G$ receptors in various immune cells including natural killer cells, macrophages, and neutrophils [24]. It has been well documented that the change of methylation levels in the extracellular domain of FCGR2A influences the ability of this receptor to bind to human IgG2 [25, 26].

Previous studies have identified FCGR2A as the susceptibility gene for $\mathrm{KD}[8,9,11]$. A functional polymorphism, encoding an H131R substitution (rs1801274), which is located just outside the $\mathrm{CNV}$ region of the $\mathrm{Fc}$ portion of $\mathrm{IgG}$, confers elevated risk of $\mathrm{KD}$ in various ethnic groups [11]. In addition, Omar et al. transfected HEK cells with these two haplotype variants of FCGR2A and studied the functional significance by testing their binding abilities to IgG subclasses [27]. They concluded that FCGR2A haplotype variants have different binding ability to IgG1, IgG3, and IgG4. Consistent with previous studies, we found that $\mathrm{CpG}$ sites $\mathrm{G}$ and $\mathrm{J}$ which are corresponding loci to NF-kB and Myc-Max are susceptibility loci of $\mathrm{KD}$. In addition, $\mathrm{CpG}$ sites $\mathrm{J}$ and $\mathrm{K}$ which are corresponding to Myc-Max and SP2 are associated with responsiveness to IVIG treatment. Therefore, these loci might be applied as important markers to evaluate the clinical outcomes of IVIG therapy in KD patients.

There are some limitations in this study. First, we focused on investigating the potential $\mathrm{CpG}$ site on FCGR2A gene promoter region within a $500 \mathrm{bps}$ region. However, other 
TABLE 4: Possible transcription factor binding sites identified by JASPAR.

\begin{tabular}{lccccc}
\hline Transcription factor & Accession $^{\mathrm{a}}$ & Score & Relative score $^{\mathrm{b}}$ & Binding site sequence & Corresponding CpG site $^{\mathrm{c}}$ \\
\hline SP1 & P08047 & 7.430 & 0.87 & GCTCTGCCTCC & TTCTCCT \\
NFATC2 & Q13469 & 6.016 & 0.80 & TTTTGT/CCGTGT & I \\
SOX10 & NP_008872 & 6.636 & 0.90 & GGGGTTTCAC & G $^{*}$ \\
NF-kB & NP_003989 & 9.015 & 0.85 & CAGGATGGTCTC & I \\
YY1 & P25490 & 8.084 & 0.84 & ATCTCCTGACC & $\mathrm{J}^{*}$ \\
USF1 & BAA76541 & 5.780 & 0.85 & GACCTCGTGAT & $\mathrm{K}^{*}$ \\
Myc-Max & AAH36092 & 8.449 & 0.81 & CACCCGCCTTGGCCT & \\
SP2 & Q02086 & 6.046 & 0.81 & & \\
\hline
\end{tabular}

${ }^{*}$ The corresponding CpG sites reached the statistical significance in Table 2 or Table 3.

${ }^{a}$ Accession number is the NCBI protein accession number.

${ }^{\mathrm{b}}$ The relative score is provided by the JASPAR according to the similarity of motif sequence.

${ }^{c}$ The sequence in our study is listed as follows:

TGCAAGCTCTGCCTCCCG ${ }^{\mathrm{A}}$ GGTTCACG $^{\mathrm{B}}$ CCATTCTCCTGCCTCAGCCTCCCG $^{\mathrm{C}}$ AGTAGCTGGGACTATCTGCCACCG $^{\mathrm{D}}$ CG $^{\mathrm{E}}$ CCCG $^{\mathrm{F}}$ GCTAAATTTTTTTTGTATTATTAGTAGAGACG ${ }^{\mathrm{G}}$ GGGTTTCACCG $^{\mathrm{H}}$ TGTTAGCCAGGATGGTCTCG $^{\mathrm{I}}$ ATCTCCTGACCTCG $^{\mathrm{J}}$

TGATCCACCCG ${ }^{\mathrm{K}}$ CCTTGGCCTCCCAAAG.

The sites marked as A-K are the methylation loci.

important $\mathrm{CpG}$ sites beyond this region were not included in our study. Therefore, extending the coverage on FCGR2A region might be necessary. Second, the SNPs or haplotypes within this promoter region could potentially influence the binding affinity of transcriptional factors such as SP2 or NF-kB; thus, confirmations of KD patients' genotypes are required. Third, the study sample size was relatively small; thus a larger sample size is necessary for a better understanding of this disease.

JASPAR is a conventional tool for predicting TFBSs and TFs. This database employs the optimized position weight matrices (PWM) for the scoring system and provides flexible scanning options to avoid the false-positive results. We also tried to use some other motif prediction programs, such as PROMSCAN [28] and TFSFAC [29]; however, the results were varied from different database. We attribute this to the different algorithms of the scoring system. Therefore, biological validations to confirm the computational prediction will be helpful to improve the study accuracy.

In summary, our study indicated a significant association between the promoter methylation of FCGR2A, susceptibility of Kawasaki disease, and therapeutic outcomes of IVIG treatment. The methylation levels of CpG sites of FCGR2A gene promoter can be important markers for optimizing IVIG therapy.

\section{Conflict of Interests}

The authors declare that there is no conflict of interests regarding the publication of this paper.

\section{Acknowledgments}

We thank Dr Chieh-Hu Lu for his support of bioinformatics analysis. This study was supported by grants from the National Science Council (NSC101-2320-B038-029-MY3; NSC 102-2314-B-182-053-MY3), Taiwan, Republic of China
(ROC) and a grant from the Chang Gung Memorial Hospital (CMRPG8C1081 and CMRPG8B0211) to Dr. Wei-Chiao Chang and Dr. Ho-Chang Kuo.

\section{References}

[1] J. W. Newburger, M. Takahashi, M. A. Gerber et al., "Diagnosis, treatment, and long-term management of Kawasaki disease: a statement for health professionals from the Committee on Rheumatic Fever, Endocarditis and Kawasaki Disease, Council on Cardiovascular Disease in the Young, American Heart Association," Circulation, vol. 110, no. 17, pp. 2747-2771, 2004.

[2] Y. Hiroshi, Y. Mayumi, O. Izumi, N. Yosikazu, and Z. Tuohong, "Thirty-year-observation of the incidence rate of Kawasaki disease in Japan," Pediatric Research, vol. 53, p. 158, 2003.

[3] A. Harnden, B. Alves, and A. Sheikh, "Rising incidence of Kawasaki disease in England: analysis of hospital admission data," British Medical Journal, vol. 324, no. 7351, pp. 1424-1425, 2002.

[4] S. Bayers, S. T. Shulman, and A. S. Paller, "Kawasaki disease: part II. Complications and treatment," Journal of the American Academy of Dermatology, vol. 69, no. 4, pp. 513.e1-513.e8, 2013.

[5] S. Bayers, S. T. Shulman, and A. S. Paller, "Kawasaki disease: part I. Diagnosis, clinical features, and pathogenesis," Journal of the American Academy of Dermatology, vol. 69, no. 4, pp. 501.el501.e11, 2013.

[6] R. Uehara, M. Yashiro, Y. Nakamura, and H. Yanagawa, "Kawasaki disease in parents and children," Acta Paediatrica, vol. 92, no. 6, pp. 694-697, 2003.

[7] Y. Onouchi, T. Gunji, J. C. Burns et al., "ITPKC functional polymorphism associated with Kawasaki disease susceptibility and formation of coronary artery aneurysms," Nature Genetics, vol. 40, no. 1, pp. 35-42, 2008.

[8] Y. Onouchi, K. Ozaki, J. C. Burns et al., "A genome-wide association study identifies three new risk loci for Kawasaki disease," Nature Genetics, vol. 44, no. 5, pp. 517-521, 2012.

[9] Y. C. Lee, H. C. Kuo, J. S. Chang et al., “Two new susceptibility loci for Kawasaki disease identified through genome-wide 
association analysis," Nature Genetics, vol. 44, no. 5, pp. 522$525,2012$.

[10] H.-C. Kuo and W.-C. Chang, "Genetic polymorphisms in Kawasaki disease," Acta Pharmacologica Sinica, vol. 32, no. 10, pp. 1193-1198, 2011.

[11] C. C. Khor, S. Davila, W. B. Breunis et al., "Genome-wide association study identifies FCGR2A as a susceptibility locus for Kawasaki disease," Nature Genetics, vol. 43, no. 12, pp. 1241-1246, 2011.

[12] H.-C. Kuo, K. D. Yang, W.-C. Chang, L.-P. Ger, and K.-S. Hsieh, "Kawasaki disease: an update on diagnosis and treatment," Pediatrics and Neonatology, vol. 53, no. 1, pp. 4-11, 2012.

[13] H.-C. Kuo, K. D. Yang, S.-H. Juo et al., "Itpkc single nucleotide polymorphism associated with the kawasaki disease in a Taiwanese population," PLoS ONE, vol. 6, no. 4, Article ID e17370, 2011.

[14] H.-C. Kuo, Y. Onouchi, Y.-W. Hsu et al., "Polymorphisms of transforming growth factor-B signaling pathway and Kawasaki disease in the Taiwanese population," Journal of Human Genetics, vol. 56, no. 12, pp. 840-845, 2011.

[15] H.-C. Kuo, H.-R. Yu, S.-H. H. Juo et al., "CASP3 gene singlenucleotide polymorphism (rs72689236) and Kawasaki disease in Taiwanese children," Journal of Human Genetics, vol. 56, no. 2, pp. 161-165, 2011.

[16] D. Fradin, S. Le Fur, C. Mille et al., "Association of the CpG methylation pattern of the proximal insulin gene promoter with type 1 diabetes," PLoS ONE, vol. 7, no. 5, Article ID e36278, 2012.

[17] T. N. Hasan, B. Leena Grace, G. Shafi, and R. Syed, "Association of BRCA1 promoter methylation with rs11655505 (c.2265C>T) variants and decreased gene expression in sporadic breast cancer," Clinical and Translational Oncology, vol. 15, no. 7, pp. 555-562, 2013.

[18] H. C. Kuo, K. D. Yang, C. D. Liang et al., "The relationship of eosinophilia to intravenous immunoglobulin treatment failure in Kawasaki disease," Pediatric Allergy and Immunology, vol. 18, no. 4, pp. 354-359, 2007.

[19] A. Sandelin, W. Alkema, P. Engström, W. W. Wasserman, and B. Lenhard, "JASPAR: an open-access database for eukaryotic transcription factor binding profiles," Nucleic Acids Research, vol. 32, pp. D91-D94, 2004.

[20] G. D. Stormo, "DNA binding sites: representation and discovery," Bioinformatics, vol. 16, no. 1, pp. 16-23, 2000.

[21] B. Lenhard, A. Sandelin, L. Mendoza, P. Engström, N. Jareborg, and W. W. Wasserman, "Identification of conserved regulatory elements by comparative genome analysis," Journal of Biology, vol. 2, no. 2, article 13, 2003.

[22] S. J. Chamberlain and M. Lalande, "Neurodevelopmental disorders involving genomic imprinting at human chromosome 15q11-q13," Neurobiology of Disease, vol. 39, no. 1, pp. 13-20, 2010.

[23] Z. Wang, Y. Zheng, C. Hou et al., "DNA methylation impairs TLR9 induced Foxp3 expression by attenuating IRF-7 binding activity in fulminant type 1 diabetes," Journal of Autoimmunity, vol. 41, pp. 50-59, 2013.

[24] T. Takai, "Roles of Fc receptors in autoimmunity," Nature Reviews Immunology, vol. 2, no. 8, pp. 580-592, 2002.

[25] P. A. M. Warmerdam, J. G. J. van de Winkel, A. Vlug, N. A. C. Westerdaal, and P. J. A. Capel, "A single amino acid in the second Ig-like domain of the human $\mathrm{Fc} \gamma$ receptor II is critical for human IgG2 binding," Journal of Immunology, vol. 147, no. 4, pp. 1338-1343, 1991.
[26] M. R. Clark, S. G. Stuart, R. P. Kimberly, P. A. Ory, and I. M. Goldstein, "A single amino acid distinguishes the highresponder from the low-responder form of Fc receptor II on human monocytes," European Journal of Immunology, vol. 21, no. 8, pp. 1911-1916, 1991.

[27] A. H. Omar, H. Shibata, M. Yasunami et al., "The rs150311303 polymorphism in Fc $\gamma$ RIIa enhances IgG binding capacity," Scandinavian Journal of Immunology, vol. 76, no. 2, pp. 167-174, 2012.

[28] D. S. Prestridge, "Predicting Pol II promoter sequences using transcription factor binding sites," Journal of Molecular Biology, vol. 249, no. 5, pp. 923-932, 1995.

[29] T. Heinemeyer, E. Wingender, I. Reuter et al., "Databases on transcriptional regulation: TRANSFAC, TRRD and COMPEL," Nucleic Acids Research, vol. 26, no. 1, pp. 362-367, 1998. 


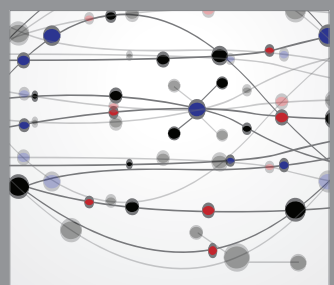

The Scientific World Journal
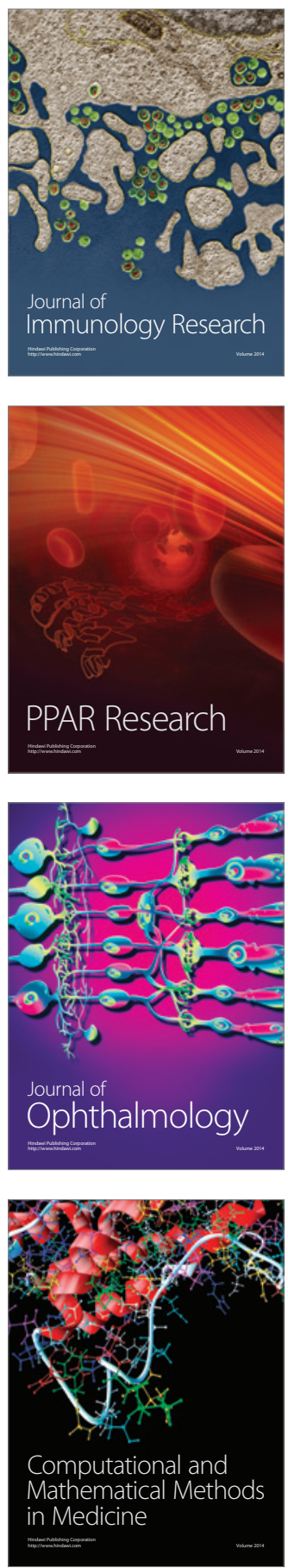

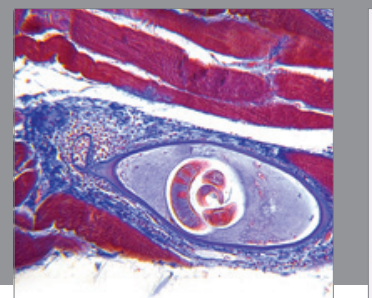

Gastroenterology

Research and Practice
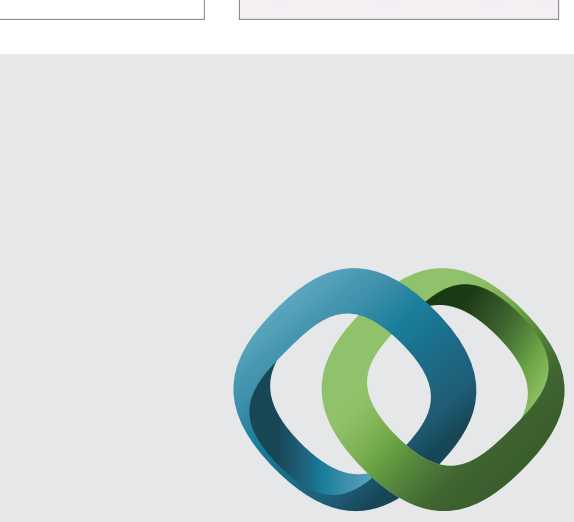

\section{Hindawi}

Submit your manuscripts at

http://www.hindawi.com
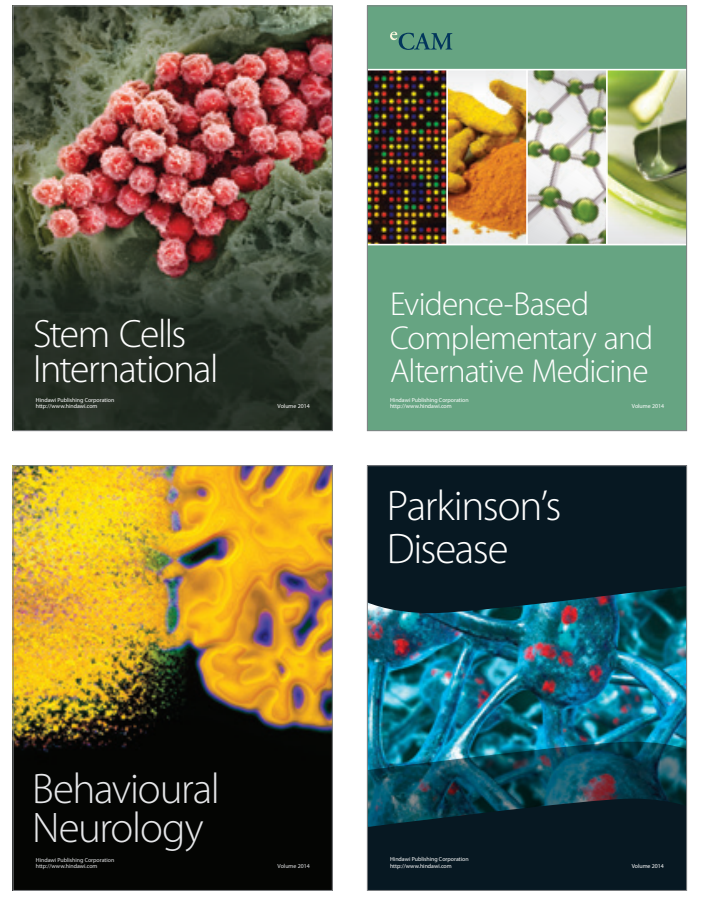
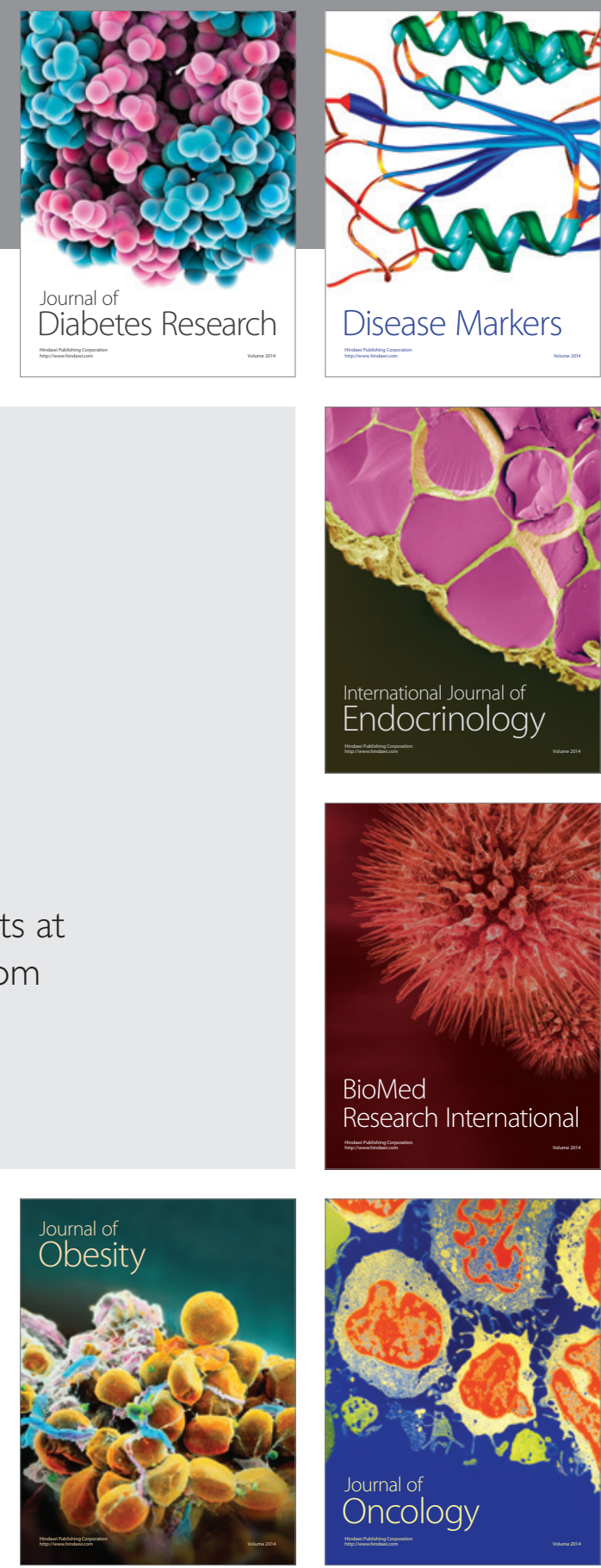

Disease Markers
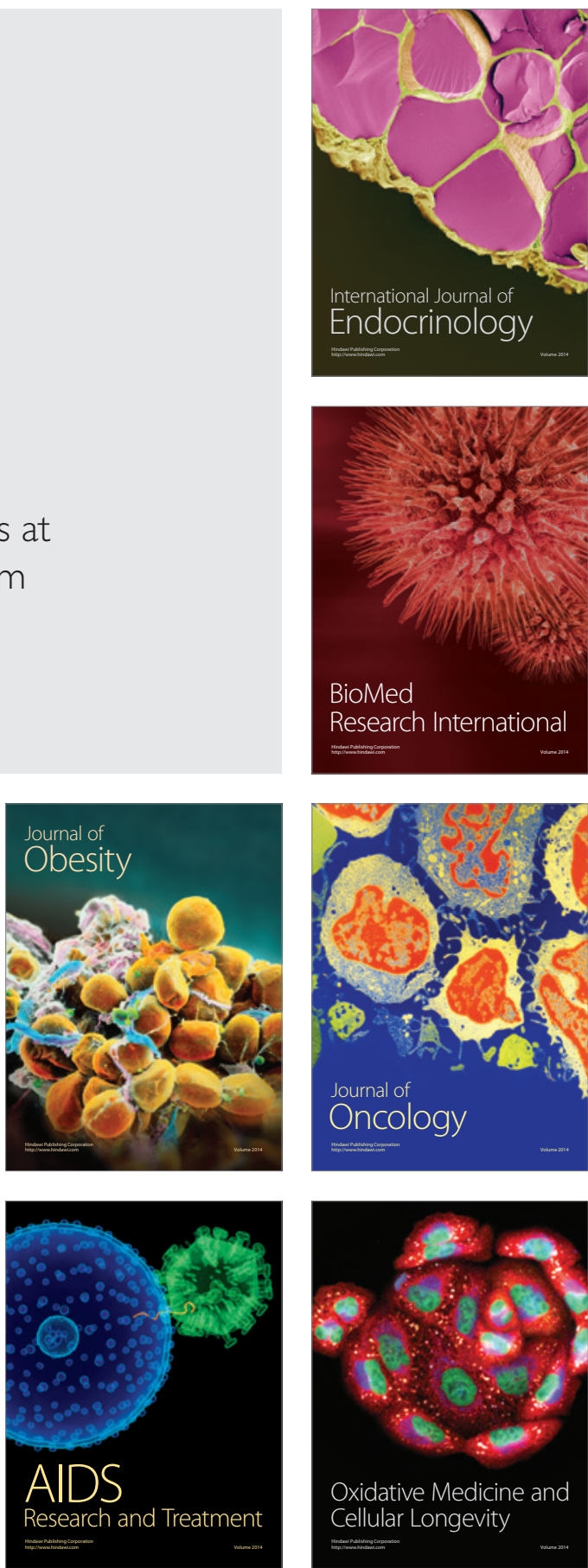